\title{
Mitigation of Lightning Surge Stresses in the High Voltage Windings of Distribution Transformers by Introducing an Electrostatic Shield
}

\author{
W. D. A. S. Wijayapala and H. S. C. Karunananda
}

\begin{abstract}
The Medium Voltage (MV) Electricity Distribution Networks are frequently exposed to lightning and it is the main suspected reason for distribution transformer failures. Appreciable number of transformer failures occur due to insulation failures notably at points near line ends. The short rise time of a surge prompted by a lightning impulse can cause deterioration in the insulation and ultimately lead to a dielectric breakdown. The voltage distribution along transformer High Voltage (HV) winding becomes non-linear under surge conditions due to the capacitive-inductive currents. This research focuses on the possibility of neutralizing this effect with the aid of an electrostatic shield inserted in to the HV winding.
\end{abstract}

Comparison of the behaviour of the winding under surge conditions with and without electrostatic shield requires appropriate transient models suitable for simulations. The necessary calculations required for this purpose were carried out and the proposed models are presented. The simulations were carried out with PSCAD software and the results are included in this paper. The oscillatory nature due to the combined effect of capacitors and inductors is observed in the wave tail region and some of the peaks created have potentials exceeding the peak potential of the surge waveform as well.

The proposed transformer model have a definite positive effect compared with the available model as the results obtained by simulations clearly reveal the reduction of the stresses on the transformer HV windings when a shield is used.

Key words: $\quad$ transformer, lightning surge, electrostatic shield, capacitance.

\section{Introduction}

\subsection{Background}

Medium Voltage (MV) Distribution Network of Ceylon Electricity Board (CEB) consists of about $29,000 \mathrm{~km}$ of $33 \mathrm{kV}$ and $11 \mathrm{kV}$ lines which spread all over the country feeding about 25,000 distribution substations. Electricity consumers expect an uninterrupted, high quality power supply. Therefore the engineers of CEB carry out a lot of projects and researches focusing on reliability improvements[1].

As the consumers are dispersed over the entire island, overhead transmission lines are running long distances through jungles, paddy fields, valleys, hills, urban and rural areas. Thus these lines are frequently exposed to lightning. Lightning strikes on medium voltage distribution lines cause power interruptions and damages to line equipment. Damage to a distribution transformer obviously becomes the costliest to the utility as well as to the consumers who experience a long interruption. The main suspected reason for transformer failures is noted as lightning according to transformer failure reports. Monthly transformer failure data of CEB Region 3 and the average monthly thunder days [2] also reveal the relationship between them.

Figure 1 represents monthly average thunder days experienced by meteorological stations located inside CEB Region 3 boundaries [2] and Figure 2 represents number of transformers belonging to CEB Region 3 failed in each month of 2010 - 2012 period.

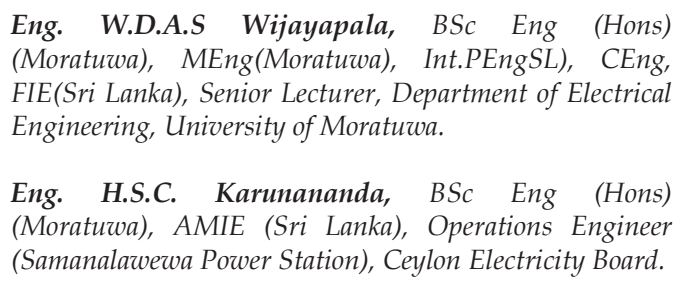

Eng. W.D.A.S Wijayapala, BSc Eng (Hons) (Moratuwa), MEng(Moratuwa), Int.PEngSL), CEng, FIE(Sri Lanka), Senior Lecturer, Department of Electrical Engineering, University of Moratuwa.

Eng. H.S.C. Karunananda, BSc Eng (Hons) (Moratuwa), AMIE (Sri Lanka), Operations Engineer (Samanalawewa Power Station), Ceylon Electricity Board. 


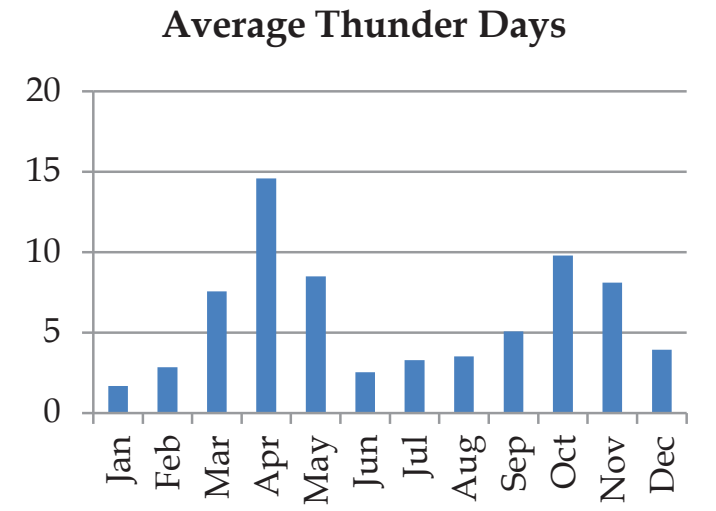

Figure 1 - Monthly average thunder days experienced in meteorological stations located inside CEB Region 3.

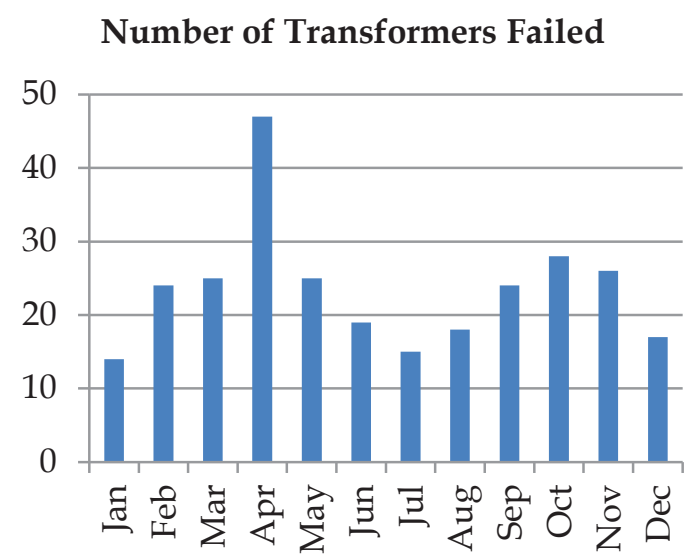

Figure 2 - Number of transformers failed in each month of 2010-2012, CEB Region 3.

\subsection{Importance of the study}

CEB uses distribution transformers with capacities of 100, 160, 400, 630, 800 and 1000 kVA and spends about 0.8 million LKR to purchase a $100 \mathrm{kVA}$ transformer. Apart from the direct material cost incurred by the failure of a transformer, there is a large hidden cost such as labor cost, transport cost, management cost and the revenue loss due to outages.

CEB medium voltage network is frequently attacked by lightning strikes and when such happens near a distribution transformer a surge is applied on the primary winding of the transformer. The sudden activity of high stress can lead to a dielectric breakdown. The severity of this process depends on several factors, such as the magnitude of the surge current. An appreciable number of transformer failures occur due to insulation failures notably at points near line ends. Surge arrestors are used to protect transformers against surges but their performance diminishes with time depending on its quality, number of strokes handled, environmental conditions etc. Poor quality surge arrestors will not function properly and may activate after the rise time of the surge waveform. It can be commonly seen that the arrestor's interconnection earth leads are not having enough cross section to handle a lighting surge of $10 \mathrm{kA}$ magnitude as stipulated in the specifications [1].

Research work on surge arrestors, arcing gaps, shielded overhead Medium Voltage (MV) and Low Voltage (LV) lines have been carried out to reduce the lightning risk on transformers, but attention has not adequately been paid on improving the lightning withstand capability of transformers. This study is focused on designing $\mathrm{HV}$ winding with appropriate electrostatic shields to reduce transformer failures by improving surge withstand capability.

The voltage distribution along transformer HV winding is linear under power frequency conditions, but it becomes non-linear under surge conditions and the potential difference between two sections or layers near the line end becomes much higher compared with the desired linear distribution. High stresses can create conditions favorable for a dielectric breakdown.

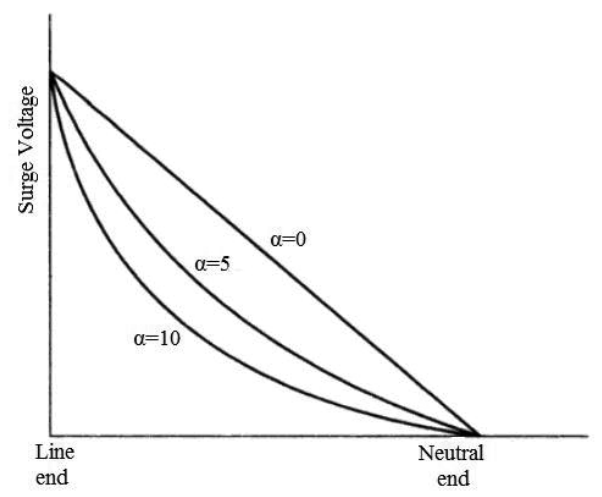

Figure 3 - Surge voltage distribution along transformer $\mathrm{HV}$ winding

The voltage $\mathrm{v}$, at a point in a single layer winding at a distancex from the neutral is given by [3],

$$
\mathrm{v}=\frac{\mathrm{V} \cosh (\mathrm{a} / l) \mathrm{x}}{\cosh \mathrm{a}}
$$

Where, $\mathrm{a}=\sqrt{ }\left(\mathrm{C}_{\mathrm{g}} / \mathrm{C}_{\mathrm{w}}\right) \ldots(2)$

$\mathrm{C}_{\mathrm{g}}=$ capacitance to earth

$\mathrm{C}_{\mathrm{W}}=$ series capacitance

$l=$ height of the winding 
The concentration of the voltage is governed by the factor $a$. Insulation failures at points near line ends can be reduced by lowering this factor. The transient voltage distribution along the winding is shown in Figure 3. The nonlinear nature of the surge voltage distribution along transformer winding increases with increasing $a$ and a uniform surge voltage distribution exist if $a$ is maintained at zero [4].

The outcome of this study was to design a 33 $\mathrm{kV}$ layer type transformer $\mathrm{HV}$ winding with reduced stresses, with the aid of an electrostatic shield.

\subsection{Objectives of the study}

The objectives of this study are,

1. To study constructional features of existing transformers which are of layer type and develop a model suitable for simulation.

2. To propose a simulation model when shields are used and analyse the surge waveforms and voltage distribution.

3. To investigate the behaviour of the shield when a chopped waveform is applied.

\section{Calculations for Standard HV Winding}

The transformer windings respond to surge voltages as a system of capacitance and inductance network [4]. The resistance of HV windings is also not negligible. Therefore it is worth calculating capacitances, inductances and resistances to propose suitable simulation models.

A $160 \mathrm{kVA}, \quad 33 / 0.415 \mathrm{kV}$ layer type transformer, manufactured by Lanka Transformers Pvt Ltd (LTL), was selected for this research. The representation of inductances, resistances and capacitances of this transformer is shown in Figure 9.

\subsection{Inductance and resistance calculations}

Inductance and resistance of the HV winding were calculated using data available on test reports and found as $2.02 \mathrm{H}$ and $165.9 \Omega$ respectively.

\subsection{Capacitance calculation \\ 2.2.1Resultant relative calculation \\ permittivity}

Capacitances exist between adjacent layers, adjacent turns, turns and tank, turns and core etc. The spaces between these surfaces are occupied by different types of insulating materials. Therefore calculation of resultant permittivity is important for determination of the capacitances.

The resultant relative permittivity $\left(\varepsilon_{\mathrm{r}}\right)$ of a space occupied by layers of different types of materials can be obtained by following formula, where $t_{1}, t_{2}, t_{3} \ldots$ and $\varepsilon_{1}, \varepsilon_{2}, \varepsilon_{3} \ldots$ are thicknesses and respective relative permittivities of different types of materials.

$\mathrm{t} / \varepsilon_{\mathrm{r}}=\mathrm{t}_{1} / \varepsilon_{1}+\mathrm{t}_{2} / \varepsilon_{2}+\mathrm{t}_{3} / \varepsilon_{3}+$

This equation is for parallel plate model but it can be approximated for cylindrical model as the radius of curvature of layers of winding are large.

The relative permittivity corresponding to all layer-layer insulation is not the same due to the different spacing and dielectric materials used. There is a cooling duct making the $8^{\text {th }}$ and $9^{\text {th }}$ layers apart by $4.5 \mathrm{~mm}$ and the respective relative permittivity was considered as that of transformer oil $\left(\varepsilon_{\text {oil }}=2.2\right)$, as it is mainly filled with oil. Resultant relative permittivities corresponding to spaces between other layers were considered as either 3.34 or 3.35 .

\subsubsection{Layer to Layer capacitance}

The winding structure can be modelled as a coaxial cylindrical capacitor, or since the radius of curvature is large, as a parallel plate capacitor [5]. Cylindrical capacitor model is selected to calculate layer-layer capacitance in this study and the static layer-layer capacitance $\left(\mathrm{C}_{1-1}\right)$ is obtained by the following equation [5],

$$
C_{1-1}=\varepsilon_{0} \varepsilon_{\mathrm{r} .} \frac{2 \Pi h}{\ln \{1+(\mathrm{d} / \mathrm{r})\}}
$$

Where,

$\mathrm{h}=$ Height of the layer

$\mathrm{r}=$ Curvature radius

$\mathrm{d}=$ Effective distance between two layers 


\subsubsection{Effective distance between two layers}

The distance between two adjacent layers plays a decisive role in the calculation of the capacitance. In general, this is not two times the insulation thickness, as one can easily and erroneously assume. The effective distance between two layers can be calculated with the following empirical formula that accounts for the actual mutual position between windings which are usually not perfectly aligned [5].

$$
d=1.26 d_{e}-1.15 d_{i}+d_{l 1}
$$

Where, $d_{e}$ and $d_{i}$ are external \& internal wire diameters. $d_{l l}$ is the layer insulation thickness.

\subsubsection{Effect of adjacent layers}

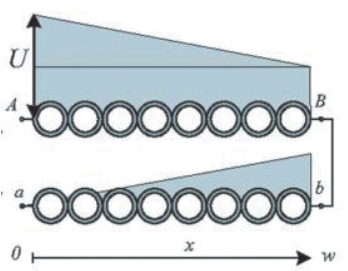

a)

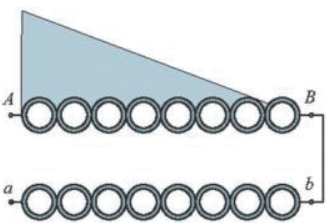

b)
Figure 4 - Potential distribution along the turns of two adjacent layers

The expression of the static layer-layer capacitance is derived under the assumptions of uniform charge distribution over the conductors of each layer and each layer is disconnected from adjacent layers at the ends. However, due to the connection among terminals of adjacent layers, the potential distribution along the turns varies and consequently so does the value of the layer-to layer capacitance [5].

In Figure 4 (a), the potential distribution along the turns of two adjacent layers is shown with respect to point "a" and Figure 4 (b) represents the potential distribution along the turns of layer $A B$ with respect to layer $a b$. When the stored electrostatic energy along the layers is considered, the effective layer-layer capacitance $\left(C_{11}\right)$ becomes $1 / 3$ of the static layer-layer capacitance [5].

The layer to layer capacitance corresponding to each layer is not the same due to the different relative permittivity, different spacing, different curvature radii of the layers and outermost layer being partially filled. The layer to layer capacitances calculated by repeating the above procedure are given in Table1.
Table 1 - Effective layer to layer capacitances

\begin{tabular}{|c|c|c|}
\hline \multirow{2}{*}{ Capacitance } & \multicolumn{2}{|c|}{ Layer to Layer Capacitance } \\
\cline { 2 - 3 } & Static $(\mathrm{pF})$ & Effective $(\mathrm{pF})$ \\
\hline L01 \& L02 & 6471 & 2157 \\
\hline L02 \& L03 & 8998 & 3000 \\
\hline L03 \& L04 & 9098 & 3033 \\
\hline L04 \& L05 & 9198 & 3066 \\
\hline L05 \& L06 & 9298 & 3099 \\
\hline L06 \& L07 & 9398 & 3133 \\
\hline L07 \& L08 & 9498 & 3166 \\
\hline L08 \& L09 & 937 & 312 \\
\hline L09 \& L10 & 9993 & 3331 \\
\hline L10 \& L11 & 10093 & 3364 \\
\hline L11 \& L12 & 10193 & 3398 \\
\hline L12 \& L13 & 10293 & 3431 \\
\hline L13 \& L14 & 10393 & 3464 \\
\hline L14 \& L15 & 7644 & 2548 \\
\hline L15 \& L16 & 7730 & 2577 \\
\hline L16 \& L17 & 4541 & 1514 \\
\hline
\end{tabular}

\subsubsection{Turn to Turn capacitance}

The capacitance between two adjacent turns of a layer per unit length can be calculated using the following formula [5].

$$
\mathrm{C}_{\mathrm{tt}, \mathrm{m}}=\varepsilon_{\mathrm{eq}} \theta \mathrm{e}^{(\mathrm{de} / \mathrm{di})}+\varepsilon_{0}\{\cot (\theta / 2)-\cot (\Pi / 12)\} \ldots
$$

Where

$$
\theta=\operatorname{Cos}^{-1}\left\{1-\varepsilon_{\mathrm{eq}}{ }^{-1} \ln \left(\mathrm{d}_{\mathrm{e}} / \mathrm{d}_{\mathrm{i}}\right\}\right.
$$

$\mathrm{d}_{\mathrm{e}} \quad=$ External diameter of conductor

$\mathrm{d}_{\mathrm{i}} \quad=$ Internal diameter of conductor

$\mathrm{C}_{\mathrm{t} t, \mathrm{~m}}=$ Turn to turn capacitance per unit length

The series capacitances of each layer are calculated considering turn lengths and number of turns in a layer. The obtained values by similar calculations are given in Table 2. 
Table 2 - Turn to turn capacitance of layers

\begin{tabular}{|c|c|c|}
\hline $\begin{array}{c}\text { Layer } \\
\text { No }\end{array}$ & $\begin{array}{c}\text { Turn to Turn } \\
\text { Capacitance }(\mathrm{pF})\end{array}$ & $\begin{array}{c}\text { Series Capacitance } \\
\text { of a Layer }(\mathrm{pF})\end{array}$ \\
\hline 01 & 53.08 & 0.169 \\
\hline 02 & 53.79 & 0.171 \\
\hline 03 & 54.39 & 0.173 \\
\hline 04 & 54.99 & 0.175 \\
\hline 05 & 55.60 & 0.177 \\
\hline 06 & 56.20 & 0.179 \\
\hline 07 & 56.80 & 0.181 \\
\hline 08 & 57.40 & 0.183 \\
\hline 09 & 59.79 & 0.190 \\
\hline 10 & 60.39 & 0.192 \\
\hline 11 & 60.99 & 0.194 \\
\hline 12 & 61.60 & 0.196 \\
\hline 13 & 62.20 & 0.198 \\
\hline 14 & 62.80 & 0.200 \\
\hline 15 & 63.51 & 0.202 \\
\hline 16 & 64.23 & 0.205 \\
\hline 17 & 64.94 & 0.357 \\
\hline
\end{tabular}

\subsubsection{Ground capacitance calculation}

\subsubsection{Capacitance between outermost layer and tank}

The capacitance between outermost layer and tank, $\mathrm{C}_{\mathrm{lt}}$, is estimated using the cylindrical capacitor model with a reduction factor of 0.75 due to the fact that the tank is not a complete circle [6]. Since the outermost layer is not fully filled with turns, there exist a ground capacitance between tank and $16^{\text {th }}$ layer and it can similarly be calculated. The formula for calculation is given below [6].

$$
\mathrm{C}_{\mathrm{lt}}=0.75 \varepsilon_{0} \varepsilon_{\mathrm{r} . \cdot} \cdot \frac{2 \Pi \mathrm{h}}{\ln \left\{\left(\mathrm{t} / \mathrm{d}_{\mathrm{o}}\right)\right\}}
$$

Where,

$\mathrm{d}_{\mathrm{o}}=$ diameter of the inner winding

$\mathrm{t}=$ internal diameter of the tank

$\mathrm{h}=$ height of the winding
The relative permittivity was considered as that of transformer oil (2.2), as it is mainly filled with oil. The thickness of paper insulation is much smaller than the thickness of oil layer. Capacitance between outermost layer and tank and $16^{\text {th }}$ layer and tank were obtained as $48.5 \mathrm{pF}$ and $33.7 \mathrm{pF}$ respectively.

\subsubsection{Capacitance between core and end} turns

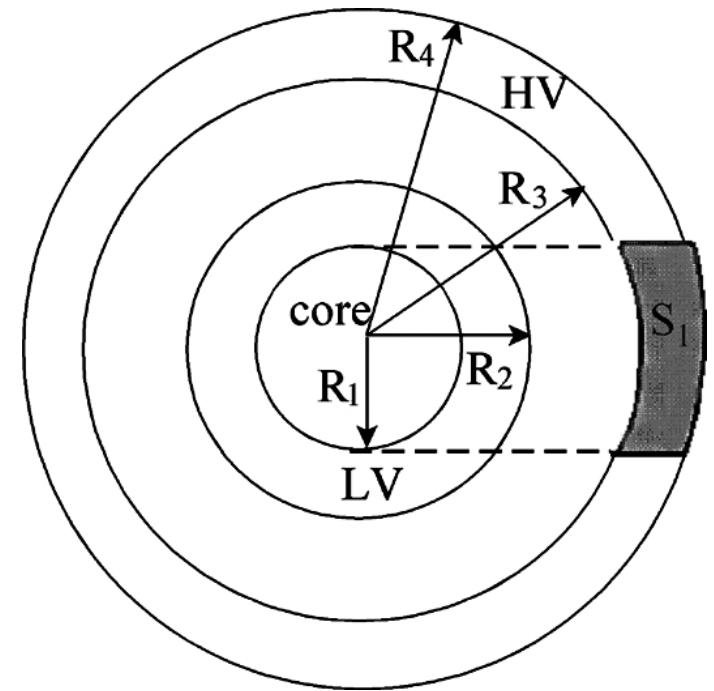

Figure 5 - Part of the HV winding surface which is located at the nearest distance from the core.

Capacitance between core and end turns of layers was calculated considering parallel plate capacitor model assuming that only the end turns of each layer contribute for capacitance of this nature [7].

The surface area " $\mathrm{S}$ " can be obtained by following formula [7].

$S=R_{4}{ }^{2} \operatorname{Sin}^{-1}\left(R_{1} / R_{4}\right)+R_{1} \sqrt{ }\left(R_{4}^{2}-R_{1}{ }^{2}\right)-R_{3}{ }^{2} \operatorname{Sin}^{-}$ ${ }^{1}\left(R_{1} / R_{3}\right)-R_{1} \sqrt{ }\left(R_{3}^{2}-R_{1}^{2}\right)$

The total capacitance between core and end turns, $C_{g g}$ can be obtained by following formula [7].

$\mathrm{C}_{\mathrm{gg}}=\varepsilon_{0} \varepsilon_{\mathrm{r}} \frac{\lceil 2 \mathrm{~S}\rceil}{\left[\mathrm{d}_{\text {top }}\right]}$

Where,

$\mathrm{d}_{\text {top }}=$ Distance between core and end turn of a layer

Accordingly $\mathrm{C}_{\mathrm{gg}}$ was calculated as $2.8 \mathrm{pF}$. 
The barrier between $\mathrm{HV}$ and $\mathrm{LV}$ windings consists of oil, paper and pressboard insulation and the resultant permittivity was calculated using equation (3). The capacitance between $\mathrm{HV}$ and $\mathrm{LV}$ windings, $\mathrm{C}_{\mathrm{HL}}$, is calculated using the cylindrical capacitor model [6].

$\mathrm{C}_{\mathrm{HL}}=\varepsilon_{0} \varepsilon_{\mathrm{r}} \cdot \frac{2 \Pi h}{\ln \left\{\left(\mathrm{d}_{\mathrm{i}} / \mathrm{d}_{\mathrm{o}}\right)\right\}}$

Where,

$\mathrm{H}=$ height of the winding

$\mathrm{d}_{\mathrm{o}}=$ Outer diameter of the LV winding

$\mathrm{d}_{\mathrm{i}}=$ Inner diameter of the HV winding

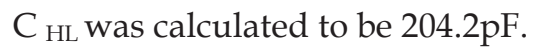

\subsubsection{Capacitance between LV winding and core}

Capacitance between LV winding and core was assumed as $300 \mathrm{pF}$ considering experimental results [8].

\section{Calculations for Shielded HV Winding}

As presented in 1.1, the surge voltage distribution along transformer winding is governed by the factor $a,\left(a=\sqrt{ } C_{g} / C_{w}\right)$ and a more uniform voltage distribution can be achieved by reducing a. The purpose of introducing an electrostatic shield in $\mathrm{HV}$ winding is to reduce a by increasing $C_{w}$.

As per the calculations shown in 2.2.4, the majority of the ground capacitance is concentrated on the outermost layer of the HV winding. Therefore the shield is inserted in between the two outermost layers (i.e. $16^{\text {th }}$ and 17th layers) as shown in Figure 6.

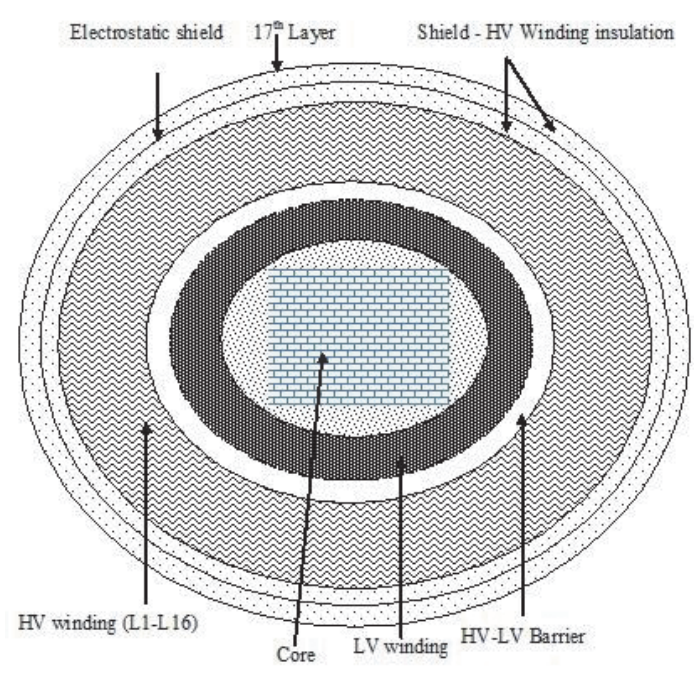

Figure 6 - Arrangement of transformer core, windings and shield

\subsection{Shield material}

The shield is not required for current handling and it shall be as thin as possible to maintain the compactness of the transformer and for minimum stray loss [9] and the cost of the material shall also be considered for minimum manufacturing cost. Aluminium foils as thin as $0.00075 \mathrm{~mm}$ are available in the market [10]. When the prices (per unit area) offered by two vendors were considered, it was found that the thinnest was not the cheapest [11], [12]. The most cost effective foil was the one having a thickness of $0.075 \mathrm{~mm}$. Prices of foils thinner than this increase with decreasing thickness due to the complexities of manufacturing processes and the prices of thicker foils increase with the increasing thickness due to high Aluminium content per unit area.

The Eddy current loss of this material shall then be evaluated to determine its suitability for the application.

\subsubsection{Eddy current loss in the shield}

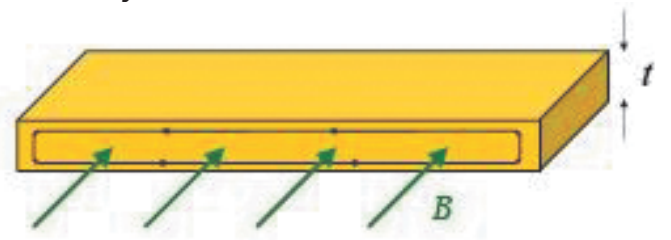

Figure 7 - Eddy current circulation inside the shield

The outermost layer (17th layer) wound on the shield creates a magnetic field inside. The magnetic flux density is obtained by the following formula [13]. 
$\mathrm{B}=\mu_{0} \mu_{\mathrm{r}} \ldots \frac{\mathrm{NI}}{l}$

Where,

$\mathrm{B}=$ Flux density

$\mu_{0}=$ Permeability of free space

$\mu_{\mathrm{r}}=$ Relative permeability of Aluminum

$\mathrm{N}=$ Number of turns in outermost layer

$\mathrm{I}=\mathrm{rms}$ full load current in the HV winding

$L=$ Height of the shield

Eddy current loss of the shield is obtained by the following formula [14].

$\mathrm{P}=\frac{4 \mathrm{AB}_{\mathrm{m}}^{2} \mathrm{f}^{2} \mathrm{t}^{3} \mathrm{k}^{2}}{3 \rho}$

Where,

$\mathrm{P}=$ Eddy current loss of the shield

$\mathrm{B}_{\mathrm{m}}=$ Peak magnetic flux density

$\mathrm{F}=$ Frequency

$\mathrm{T}=$ Thickness of the shield

$\mathrm{K}=$ Form factor $=1.11$

$\mathrm{P}=$ Resistivity of Aluminium

A $=$ Surface Area of the shield

The total eddy current loss at full load has become $4.9 \times 10^{-7} \mathrm{~W}$, which is very small and is negligible compared to the total loss at full load of the transformer selected for this study. Therefore, the Aluminum foil having a thickness of $0.075 \mathrm{~mm}$ becomes appropriate for this application.

\subsection{Capacitance calculation}

Resultant relative permittivities were calculated as presented in 2.2.1.

\subsubsection{Shield to tank capacitance}

This capacitance can be calculated using cylindrical capacitor theory as in outermost layer to tank capacitance calculation presented in 2.2.4.1 and the results are given in Table 3 [06].

Table 3 - Capacitances when the shield is present between $16^{\text {th }}$ and $17^{\text {th }}$ layers

\begin{tabular}{|l|c|c|}
\hline \multirow{2}{*}{ Capacitance } & \multicolumn{2}{|c|}{ Capacitance } \\
\cline { 2 - 3 } L16 \& Shield & 9140 & 3047 \\
\hline L17 \& Shield & 5341 & 1780 \\
\hline Tank \& Shield & N/A & 34.4 \\
\hline Tank \& L17 & N/A & 49.4 \\
\hline
\end{tabular}

\subsection{2 $16^{\text {th }}$ layer to shield capacitance}

This capacitance can be calculated using cylindrical capacitor theory as in layer to layer capacitance calculation presented in 2.2.2. The shielded $\mathrm{HV}$ winding is considered as a winding having 18 layers.

$\mathrm{C}_{1-\mathrm{l}}=\varepsilon_{0} \varepsilon_{\mathrm{r}} \ldots \frac{2 \Pi h}{\ln \{1+(\mathrm{d} / \mathrm{r})\}}$

Where,

$\mathrm{h}=$ Height of the layer

$\mathrm{r}$ = curvature radius

$\mathrm{d}=$ Distance between two layers $=t_{c}+d_{l l}$

Where $t_{c}$ is the $\mathrm{HV}$ conductor insulation thickness and $d_{l l}$ is the paper insulation thickness.

\subsubsection{7th layer to shield capacitance}

This capacitance can be calculated similar to that of $16^{\text {th }}$ layer to shield capacitance and the results are given in Table 3.

\subsubsection{7 ${ }^{\text {th }}$ Layer to tank capacitance}

This capacitance can be calculated using cylindrical capacitor theory as in outermost layer to tank capacitance calculation presented in 2.2.4.1 and the results are given in Table 3 [6].

None of the other capacitances change due to the insertion of the shield.

\subsection{Insulation of the shield}

Oil impregnated paper insulation of $0.75 \mathrm{~mm}$ thickness is proposed to have between the shield and the HV winding along the full height of the shield, similar to Layer to Layer insulation [9].

\section{Simulation and Results}

Simulation circuits were designed using the results of the calculations presented in 2 and 3 above. The purpose of the simulations is to investigate the behaviour of the transformer winding under the impact of lightning and to investigate the improvement of the surge voltage distribution when a shield is used. The input surge waveforms, simulation models and simulation results are presented below.

\subsection{The surge waveform}

A surge on a transmission system due to lightning discharge may have an extremely 
complicated waveform. For the purpose of testing of transformers, as per the IEC 60060-1, the 1.2/50 $\mu \mathrm{s}$ waveform has been formulated for the full wave impulse. It has a wave front of $1.2 \mu$ s and wave tail of $50 \mu s$.

The standard IEC waveform can be mathematically defined by following formula [15].

$$
\mathrm{V}(\mathrm{t})=\mathrm{e}^{-\mathrm{\alpha t}}-\mathrm{e}^{-\beta \mathrm{t}} \ldots(15)
$$

Where, $\alpha=0.0143$ and $\beta=4.87$ when $t$ is in microseconds.

The respective output waveform is given in Figure 8.

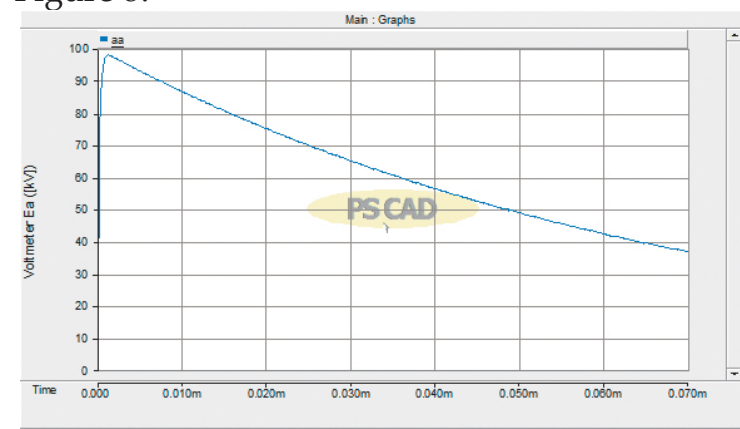

Figure 8 - Surge waveform used for simulations

\subsection{Simulation Model}

The outermost HV layer of the transformer selected for study is partially filled by turns. Since higher portion of ground capacitance is associated with the outermost layer, it is essential to distribute it along the outermost layer and analyse rather than lump all resistances, inductances and capacitances in to layers and analyse. There are 183 turns in this layer and is split in to three sections (61 turns each) as shown in Figures 9 and 11. Other layers contain 315 turns in each and these are split in to five sections (63 turns each). Resistances, inductances and capacitances in respect of each segment were calculated using the results obtained previously for individual layers. The simulation circuits were designed accordingly. All the simulation models are in single phase configuration. The points A and B as shown in Figures 9 and 11 are the highest stressed points in the configuration. The potential difference between the two points versus time was plotted for both cases, with and without electrostatic shield to assess the impact of inserting the shield.

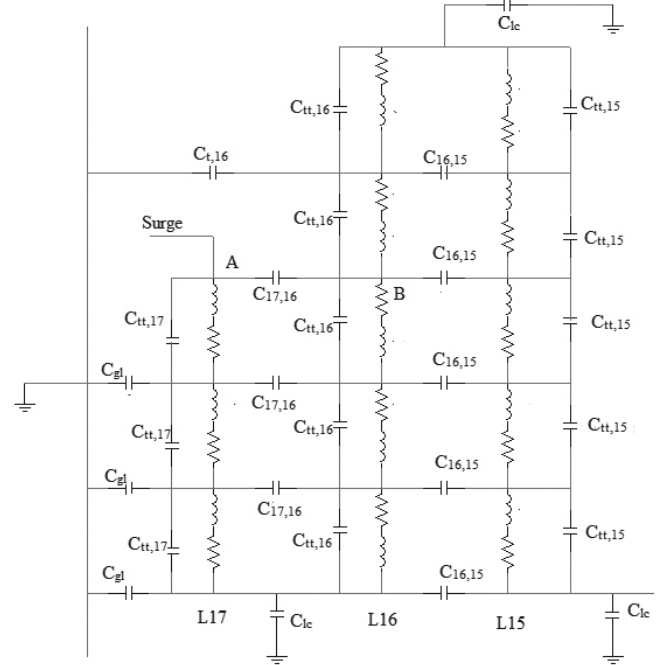

Figure 9 - Resistance, inductance and capacitance network of layer winding without electrostatic shield

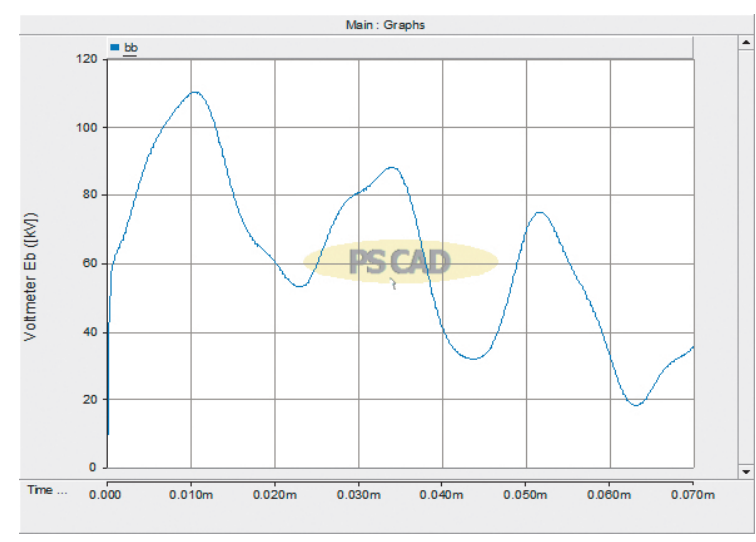

Figure 10 - The output waveform at the junction of $16^{\text {th }}$ and $17^{\text {th }}$ layers

Resistance, inductance and capacitance network of HV winding without electrostatic shield is given in Figure 9 and the output waveform at the junction of $16^{\text {th }}$ and $17^{\text {th }}$ layers is given in Figure 10. The oscillatory nature due to the combined effect of capacitors and inductors is observed in this waveform and the peak of the voltage reaches as close as 110 $\mathrm{kV}$ whereas the input surge voltage remains around $98 \mathrm{kV}$. This reveals the fact that the resistances and inductances shall also be considered during simulations of this nature and the "capacitor only" circuits are not good enough. 


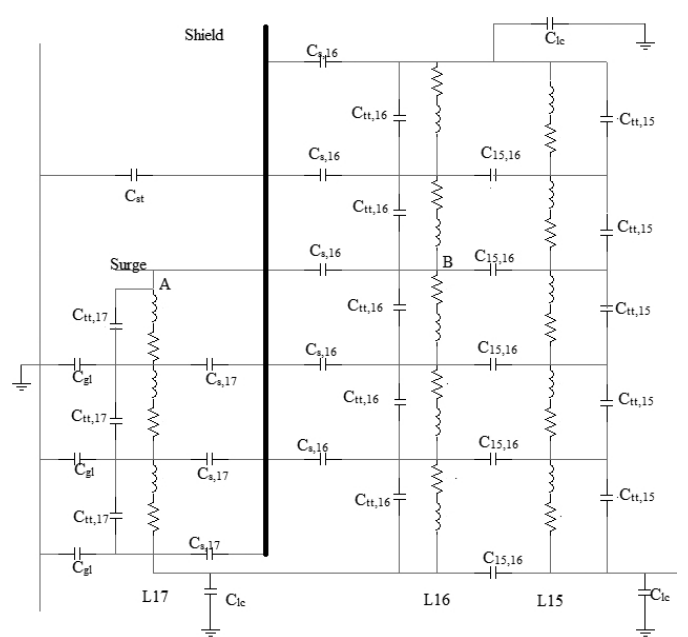

Figure 11 - Resistance, inductance and capacitance network of layer winding with electrostatic shield

The plots of potential difference between the two points $\mathrm{A}$ and $\mathrm{B}$, with and without electrostatic shield were plotted and are shown in Figure 12 and Figure 13 respectively. The two graphs are on same scale for better comparison. The improvement of voltage distribution is obvious as the stress has reduced considerably when the shield is used. The maximum stresses between the two points become $17.4 \mathrm{kV}$ without the shield and it is as low as $5.0 \mathrm{kV}$ when the shield is present.

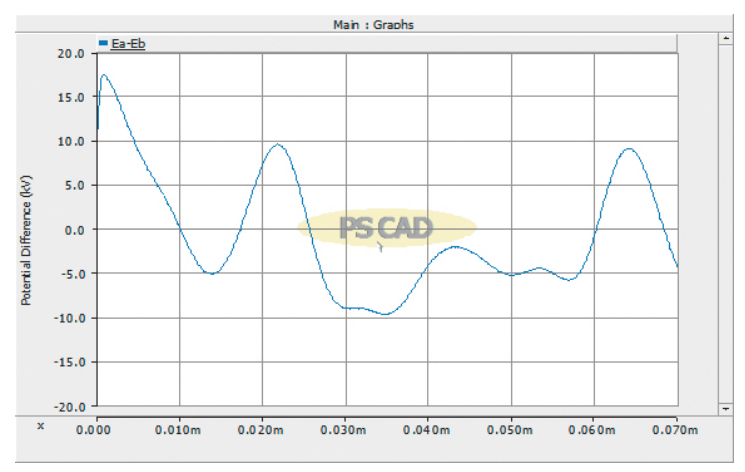

Figure 12 - Potential difference between A and $B$ without shield

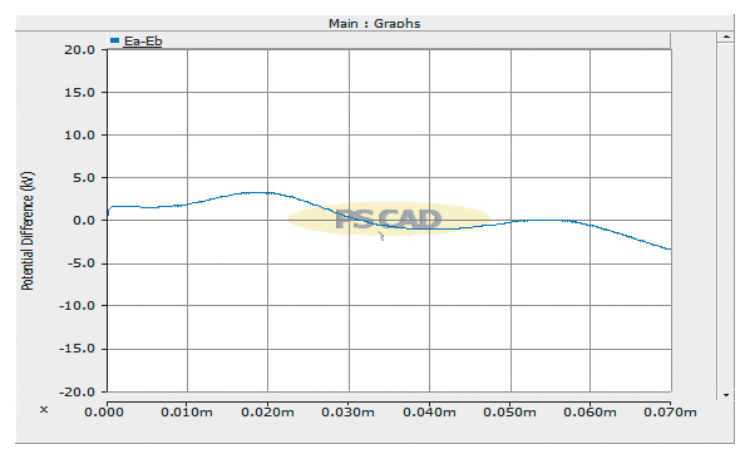

Figure 13 - Potential difference between A and $B$ with shield
4.3 Surge voltage distribution along transformer $\mathrm{HV}$ winding

The theories behind the non-linear nature of the surge voltage distribution along a single layer transformer winding were discussed in 1 above. With the winding representations and simulation models proposed in 2 and 3 above, opportunity is now available to plot the potential distributions with and without the presence of the shield, based on the simulation results.

Peak voltages observed in seven outermost layers during the wavefront period were obtained by the simulations. The surge voltage distribution was plotted against percentage winding length and is shown in Figure 14.

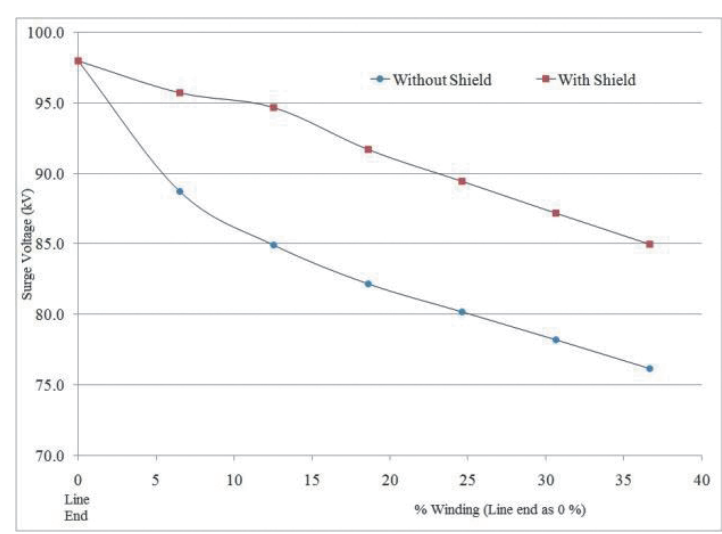

Figure 14 - Surge voltage distribution against percentage winding length

The steep drop of surge potential along the turns near the line end and the desired improvement of the potential distribution when an electrostatic shield is used are revealed by this plot.

\subsection{The chopped waveform}

Distribution transformers in CEB network are protected by surge arrestors. Arcing gaps are also present to provide additional protection. The diversion of surge current through these devices causes sudden collapse of impulse voltage and results in high rate of change of voltage in the transformer winding. This type of wave is termed as chopped wave impulse

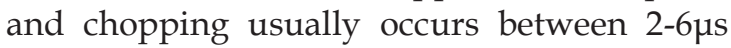
[4]. It is worth examining the behaviour of the standard and shielded winding when subjected to chopped surges due to action of surge arrestors' or arcing horns. 
The generation of the standard surge waveform was discussed in section 4.1. Waveform chopped at $1 \mu$ is used for simulations and the respective output waveform is shown in Figure 15.

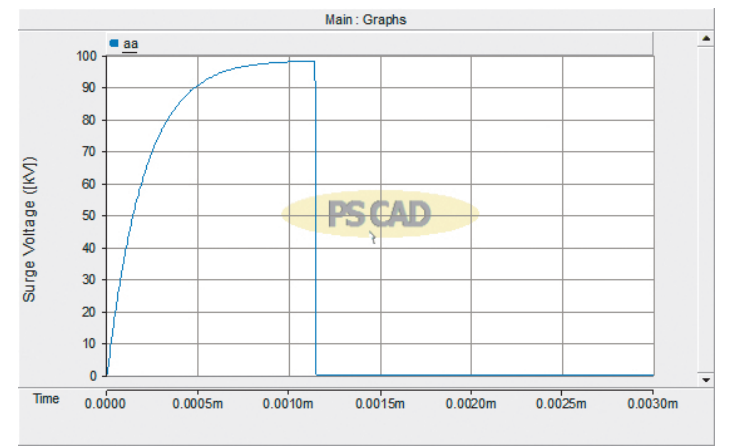

Figure 15 - Waveform chopped at 1 1s

Similar to the simulations stipulated in section 4.2 , the plots of potential difference between the two points A and B as shown in Figures 9 and 11, with and without electrostatic shield were plotted when the winding is subjected to the chopped wave are shown in Figure 16 and Figure 17 respectively. Again the two graphs are on same scale for better comparison. The improvement of voltage distribution even when a chopped waveform is applied is obvious as the stress has reduced considerably when the shield is present.

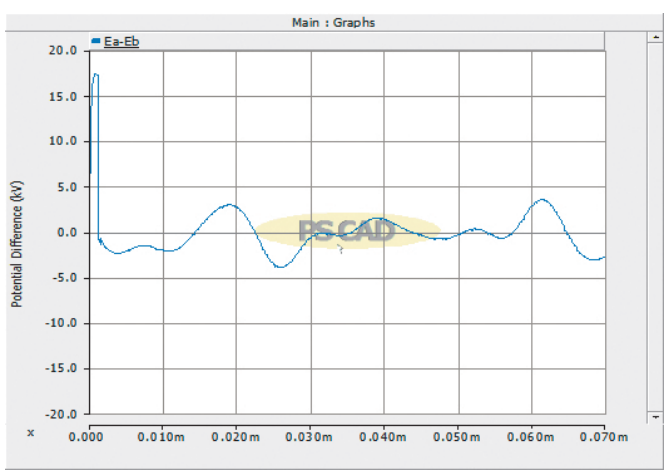

Figure 16 - Potential difference between A and B, without shield when chopped waveform is applied

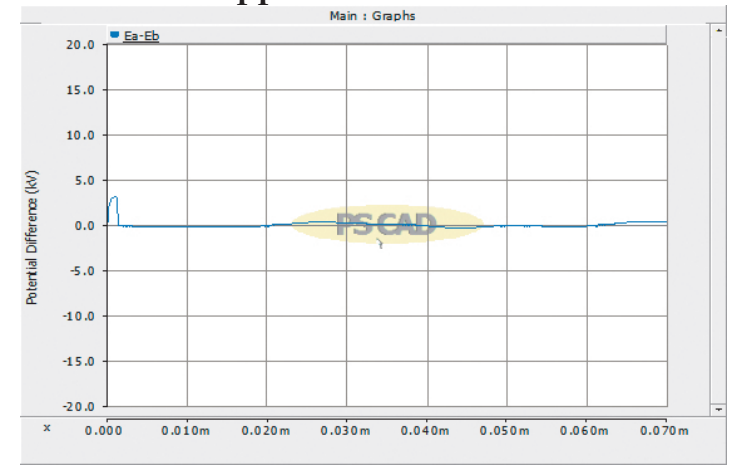

Figure 17 - Potential difference between A and $B$, with shield when chopped waveform is applied

\section{Conclusions and Recommendations}

The voltage distribution along transformer HV winding becomes non-linear under surge conditions due to the capacitive currents. The possibility of neutralizing this effect with the aid of an electrostatic shield is investigated in this research and the simulations results are positive.

The comparison of the behaviour of the winding under surge conditions with and without electrostatic shield requires appropriate transient models suitable for simulations. The necessary calculations for this purpose and the proposed models are presented in sections 2, 3 and 4 above. The simulations were carried out with PSCAD software and the results are presented in Section 4 and a reduction of surge stresses on the HV winding is observed.

The oscillatory nature due to the combined effect of capacitors and inductors is observed in the wavetail region and some of the peaks created have potentials exceeding the peak potential of the surge waveform.

The results obtained by simulations clearly reveal the improvement of the surge withstand capability of distribution transformers with the use of electrostatic shields. However experimental validation is outside the scope of this research work. Therefore it is strongly recommended to confirm the results experimentally and to manufacture transformers with electrostatic shields for higher degree of surge withstand capability.

\section{References}

[1] Wijekoon, V. B., Wijekoon, H. M., "Measures to Minimize Distribution Transformer Failures in the MV Network of Ceylon Electricity Board, Sri Lanka".

[2] "Hazard Profiles in Sri Lanka, Lightning.pdf", Internet article,

http://www.hazard.lk/Hazard\%20Profile/Li ghtning.pdf, Ministry of Disaster Management, Sri Lanka, pp. 105-123.

[3] Lucas, J. R. "High Voltage Testing", High Voltage Engineering, University of Moratuwa, pp. 155-158. 
[4] Prabhakar, M. V. and Gupta, S. K., "Windings and Insulation, Transformers", Bharat Heavy Electricals Limited, 02nd Edition, pp. 108-137, (2003).

[5] Luca Dalessandro, Fabiana Da Silveira, Kolar, Johann W., "Self Capacitance of High-Voltage Transformer", IEEE Transactions on Power Electronics, Vol. 22, No. 5, September 2007.

[6] Bjerkan, Eilert, "High Frequency Modelling of Power Transformers, Stresses \& Diagnostics", Trondheim, May 2005.

[7] Marjan Popov, Lou Van der Sluis, Rene Peter Paul Smeets, Jose Lopez Roldan, "Analysis of Very Fast Transients in Layer Type Transformer Windings", IEEE Transactions on Power Delivery, Vol.22, No.1, January 2007.

[8] Fernando, M. A. R. M., Cooray, V., "Lightning Surges at Distribution Transformer Secondary" ICIIS 2010, Jul 29-Aug 01, 2010, India.

[9] Heathcote, Martin J., “The J \& P Transformer Book, Reed Educational and Professional Publishing Ltd, 12 $2^{\text {th }}$ Edition, pp. 103-155, (1998)".

[10] "Aluminum Foil", Internet article, http://en.wikipedia.org/wiki/Aluminium_fo il.

[11] "Advent Research Materials, Aluminum Foils", Internet article,

http://www.adventrm.com/catalogue/lines. aspx?criteria $=$ materialform $\&$ materialid $=2 \&$ for $\operatorname{mid}=4$.

[12] "Good fellow On-line Interacting Catalog", Internet article,

http://www.goodfellow.com/catalogue/GF Cat4.php?ewd_token=3HaNjvvV0GzaEckGC mpUUtd7Bvawv2\&n=aWKsCOntG4bcl7SBXR hlQI0cFILQ1v.

[13] "Solenoid", Internet article, http://en.wikipedia.org/wiki/Solenoid.

[14] Lucas, J. R. "Electromagnetic Theory", University of Moratuwa, pp. 6-7.

[15] Lucas, J. R. “High Voltage Surge Generators", High Voltage Engineering, University of Moratuwa, pp. 132-145.

[16] Weerawardane, W. A. V., Wijekoon, H. M., Ceylon Electricity Board, Sri Lanka, "Analysis of Transient over Voltages in Medium Voltage Distribution Network of Ceylon Electricity Board, Sri Lanka".
[17] “Relative Permittivity - Dielectric Constant, Some common materials and relative permittivity", Internet article, http://www.engineeringtoolbox.com/relative -permittivity-d_1660.html.

[18] "Dielectric Constant, Strength, \& Loss Tangent", Internet article, http://www.rfcafe.com/ references/electrical/dielectric-constantsstrengths.htm.

[19] "Transformer Engineering Design and Practice", Internet article, http:/ /203.158.253.140/media/eBook/Engine er/Electrical/Transformer \%20Engineering \% 2 0 -

\%20Design \%20and \%20Practice/DK1232ch7.p df, pp. 277-325.

[20] JuerganBiela, and Kolar, Johann W., “Using Transformer Parasitics for Resonant Converters - A Review of the Calculation of the Stray Capacitance of Transformers", IEEE Transactions on Industry Applications, Vol. 44, No. 1, January/February 2008. 
